\title{
NUP50 is necessary for the survival of primordial germ cells in mouse embryos
}

\author{
Eunsook Park ${ }^{1,2}$, Bobae Lee ${ }^{1}$, Bruce E Clurman $^{3}$ and Keesook Lee ${ }^{1}$ \\ ${ }^{1}$ School of Biological Sciences and Technology and ${ }^{2}$ Korea Basic Science Institute Gwangju Center, Chonnam \\ National University, Gwangju 500-757, Republic of Korea and ${ }^{3}$ Divisions of Clinical Research and Human Biology, \\ Fred Hutchinson Cancer Research Center, Seattle, Washington 98109, USA
}

Correspondence should be addressed to K Lee; Email: klee@chonnam.ac.kr

\begin{abstract}
Nucleoporin $50 \mathrm{kDa}$ (NUP50), a component of the nuclear pore complex, is highly expressed in male germ cells, but its role in germ cells is largely unknown. In this study, we analyzed the expression and function of NUP50 during the embryonic development of germ cells using NUP50-deficient mice. NUP50 was expressed in germ cells of both sexes at embryonic day 15.5 (E15.5), E13.5, and E12.5. In addition, NUP50 expression was also detected in primordial germ cells (PGCs) migrating into the genital ridges at E9.5. The gonads of Nup50-/- embryos of both sexes contained few PGCs at both E11.5 and E12.5 and no developing germ cells at E15.5. The migratory PGCs in Nup50-/- embryos at E9.5 showed increased apoptosis but a normal rate of proliferation, resulting in the progressive loss of germ cells at later stages. Taken together, these results suggest that NUP50 plays an essential role in the survival of PGCs during embryonic development.

Reproduction (2016) 151 51-58
\end{abstract}

\section{Introduction}

Primordial germ cells (PGCs), which are derived from the proximal epiblast cells, are first detectable at the base of the incipient allantois at embryonic day 7.5 (E7.5) in mice (Ginsburg et al. 1990). Starting at E8.5, the majority of PGCs begin to migrate along the midline of the embryo through the hindgut and arrive at the genital ridges at E10.5 (Anderson et al. 2000). During migration, PGCs expand from $\sim 100$ cells at E8.5 to $\sim 25000$ cells at E13.5 (Tam \& Snow 1981). In the genital ridges, PGCs continue to divide several times and differentiate into male or female germ cells following sex determination of the somatic gonads (McLaren 2003).

During their migration into the genital ridges, the PGCs are regulated by the balance between survival and proliferation. Several genes that are required for PGC survival during migration have been identified. For example, a deficiency of NANOS3, a gene that is expressed in PGCs at this stage, causes the complete loss of germ cells in both sexes, suggesting it plays a role in the maintenance of the PGC population, presumably by supporting proliferation and/or suppressing apoptosis (Tsuda et al. 2003). The RNA-binding protein, dead end homolog 1 (DND1), has been shown to be required to prevent PGC death and maintain PGC numbers during their migration (Weidinger et al. 2003). In addition, Steel factor is essential for the normal proliferation and migration of PGCs and controls the death of any remaining PGCs in the midline structures during the late stages of development (Runyan et al. 2006).

The vertebrate protein nucleoporin $50 \mathrm{kDa}$ (NUP50) is a component of the nuclear pore complex (NPC) that mediates molecular transport between the nucleus and the cytoplasm (Doye \& Hurt 1997). NUP50 contains a characteristic NUP motif consisting of phenylalanine and glycine repeats and has been shown to interact with importins $\alpha$ and $\beta$ to simulate the import of proteins containing a nuclear localization signal (NLS; Lindsay et al. 2002). However, another study revealed that NUP50 functions in disassembling nuclear protein import complexes and recycling importins rather than chaperoning the import complex through the NPC (Matsuura \& Stewart 2005).

NUP50, which is weakly expressed in most tissues but is highly expressed in the developing neural tube and the adult testes, affects mouse development (Smitherman et al. 2000). NUP50 deficiency causes embryonic lethality associated with neural tube abnormalities, exencephaly, and intrauterine growth retardation. In the adult testes, NUP50 is strongly expressed in developing germ cells, particularly spermatocytes (Smitherman et al. 2000). In addition, the subcellular distribution of the NUP50 protein changes markedly during male germ cell differentiation, from an NPC like staining in spermatocytes to whole nucleus staining in spermatids and finally to a nuclear surface staining in mature spermatozoa 
(Fan et al. 1997). However, the function of NUP50 in germ cells has not yet been determined.

In this study, we investigated the expression and role of NUP50 during the embryonic development of the germ cells using NUP50-deficient (Nup50-/-) embryos. During the development of WT mouse embryos, NUP50 was expressed in both PGCs and fetal germ cells. PGCs in Nup50-/- embryos showed a high level of apoptosis but a normal rate of proliferation during the migratory and proliferative phases. Taken together, these results suggest that NUP50 is required for the survival of PGCs during their development.

\section{Materials and methods \\ Animals and sex determination}

Nup50-/- embryos were obtained by crossing Nup50+/male and female mice with the C57BL/6 background (Smitherman et al. 2000). The day at which a vaginal plug was detected was designated as E0.5. All of the animal procedures were approved by the Institutional Animal Care and Use Committee (IACUC) of Chonnam National University (permit number: 2012-44). For the sex determination of embryos, PCR was performed with gDNA using primers specific for the Smcy and Smcx genes: SMCX-1 (5'-CCGCTGCCAAATTCTTTGG-3') and SMC4-1 (5'-TGAAGCTTTTGGCTTTGAG-3') (Agulnik et al. 1994).

\section{$\beta$-galactosidase staining}

$\beta$-galactosidase ( $\beta$-gal) staining was performed as previously described (Nagy 2003). Briefly, embryos and embryonic gonads were incubated in a fixative solution containing $0.2 \%$ glutaraldehyde and washed with detergent rinse. Tissues were then stained in a staining solution containing $1 \mathrm{mg} / \mathrm{ml} \mathrm{X-gal} \mathrm{for}$ $5 \mathrm{~h}$ at $37^{\circ} \mathrm{C}$. To generate sections, embryonic gonads were postfixed in $4 \%$ formaldehyde, dehydrated, and then embedded in paraffin. The sections were counterstained with $0.1 \%$ Nuclear Fast Red.

\section{Alkaline phosphatase staining \\ PGCs in embryos were detected by alkaline phosphatase (AP) staining as previously described (Ginsburg et al. 1990). Briefly, embryos and embryonic gonads were fixed in $4 \%$ formal- dehyde. After treatment with $70 \%$ ethanol for $2 \mathrm{~h}$ at $4{ }^{\circ} \mathrm{C}$, the samples were stained with a freshly prepared AP solution for 5-10 min. The stained embryos and gonads were visualized under a dissecting microscope (Leica MZ-16).}

\section{Immunohistochemistry and immunofluorescence}

For immunohistochemistry, embryonic gonads were fixed in Bouin's solution (Sigma) and embedded in paraffin. The slides were processed for immunohistochemistry using the Histostain Plus Kit (Zymed, San Francisco, CA, USA) according to the manufacturer's instructions. After antigen retrieval processing, the sections were blocked in $10 \%$ nonimmune serum and incubated with anti-GCNA1 (a kind gift from Dr George C Enders) or anti-Müllerian inhibiting substance (anti-MIS) (Santa Cruz Biotechnology) antibody overnight at $4{ }^{\circ} \mathrm{C}$. The next day, the samples were incubated in secondary antibody and then counterstained with Mayer's hematoxylin (Thermo Scientific, Hudson, NH, USA).

For immunofluorescence, embryos and embryonic gonads were fixed in $4 \%$ formaldehyde and embedded in optical cutting temperature (OCT) compound (Leica, Bensheim, Germany). For whole-mount immunofluorescence, the embryos were fixed in $4 \%$ formaldehyde. The slides or the embryos were blocked in 2\% BSA containing $0.1 \%$ Triton $\mathrm{X}-100$ for $4 \mathrm{~h}$ at room temperature (RT) and incubated with anti-NUP50 (Smitherman et al. 2000), anti-SSEA1 (Developmental Studies Hybridoma Bank (DSHB), lowa City, IA, USA), anti-PGC7 (a kind gift from Dr Toshinobu Nakamura), antibromodeoxyuridine (anti-BrdU) (Roche), anti-cleaved caspase-3 (Cell Signaling, Beverly, MA, USA), or anti-phospho-histone $\mathrm{H} 3$ (Cell Signaling) antibody overnight at $4{ }^{\circ} \mathrm{C}$. After incubation with Alexa 488-conjugated goat anti-rabbit IgG, Alexa 488conjugated goat anti-mouse IgG + IgM, Cy3-conjugated goat anti-rabbit IgG, or Alexa 555-conjugated goat anti-rabbit IgG (Molecular Probes, Eugene, OR, USA) at RT for $1 \mathrm{~h}$, the images of labeled embryos or the slides were visualized by confocal microscopy (Leica TCS SPE). The fluorescence images were analyzed using the Leica LA SAF Software.

\section{LysoTracker staining}

Apoptotic germ cells in Nup50-/- embryos at E9.5 were detected using LysoTracker Red DND-99 (Invitrogen) in wholemount gonads as previously described (Yao et al. 2002). Briefly, dissected embryos were cultured in $500 \mu$ of DMEM medium with $1 \mu \mathrm{l}$ of LysoTracker for $30 \mathrm{~min}$. The embryos were fixed in $4 \%$ formaldehyde for $20 \mathrm{~min}$ and processed for whole-mount immunofluorescence.

\section{BrdU incorporation}

For BrdU labeling, pregnant females at E9.5 were intraperitoneally injected with $50 \mathrm{mg} / \mathrm{kg}$ BrdU (Sigma) and killed $6 \mathrm{~h}$ later. The dissected embryos were fixed in $4 \%$ formaldehyde and embedded in OCT compound.

\section{Statistical analysis}

The data are presented as the means \pm s.D. Statistical significance was calculated by two-tailed unpaired Student's $t$-test using the GraphPad Prism 5 Software (San Diego, CA, USA). For statistical analyses, $P<0.05$ was considered significant.

\section{Results}

\section{Expression of NUP50 in germ cells during embryonic development}

Previous studies have demonstrated that NUP50 is expressed in the postnatal testis during male germ cell differentiation (Fan et al. 1997, Smitherman et al. 2000). To explore the expression of NUP50 in germ cells during 
embryonic development, we performed immunofluorescence with WT embryonic gonads. NUP50 expression was detected in GCNA1-positive germ cells of both sexes at E15.5, E13.5, and E12.5 (Fig. 1A, B and C). In addition, weak NUP50 expression was observed in somatic cells of the gonads. The expression of NUP50 in PGCs and fetal germ cells was more prominent than in somatic cells and appeared to be increased at later developmental stages of germ cells. At E9.5, the time of PGC migration into the genital ridges, NUP50 was uniformly expressed in both migrating SSEA1-positive PGCs and somatic cells (Fig. 1D).

We confirmed the expression of NUP50 in embryonic gonads using Nup50+/- mice. Nup50-knockout mice, which are produced by introducing the $L a c Z$ gene under the control of the Nup50 promoter, allowed us to determine the expression of endogenous Nup50 by analyzing $\beta$-gal activity (Smitherman et al. 2000). Consistent with the results obtained from the immunofluorescence analyses, $\beta$-gal activity was detected in germ cells of the gonads, testes, and ovaries, at E15.5 (Fig. 2A). During the early stages of development, $\beta$-gal was expressed in the whole embryos as well as the area containing PGCs at E7.5 (Fig. 2B), and in whole embryos containing migrating PGCs at E8.5 (Fig. 2C). These results suggest that NUP50, which is highly expressed in germ cells of the embryonic gonads, may play a role in the establishment and development of germ cells.

\section{The development of PGCs is impaired in Nup50-/- embryos}

To investigate whether NUP50 deficiency affects the embryonic development of germ cells, we performed immunohistochemistry using an anti-GCNA1 antibody to quantify the number of germ cells in Nup50-/gonads at E15.5. Interestingly, a lack of germ cells was observed in Nup50-/- fetal testes and ovaries but not in Nup50+/- gonads (Fig. 3A). Previous studies have shown that many growth factors derived from Sertoli cells, such as stem cell factor, basic fibroblast growth factor, and leukemia inhibitory factor, are necessary for the survival and proliferation of PGCs (Dolci et al. 1991, Resnick et al. 1992). Therefore, we further examined whether the lack of germ cells in Nup50-/- fetal testes is due to a defect in Sertoli cells during gonad development. The MIS, a Sertoli cell-specific product, was expressed normally in Nup50-/- fetal testes lacking germ cells as well as in Nup50+/- fetal testes at E15.5 (Fig. 3B), indicating normal somatic development in Nup50-/- fetal testes.

In Nup50-/- embryonic gonads at both E11.5 and E12.5, the postmigratory phase of PGC development, AP-positive PGCs (Ginsburg et al. 1990) were remarkably reduced compared with Nup50+/- embryonic gonads, and few AP-positive PGCs were observed in the male and female gonads (Fig. 3C and D). The impaired
PGC development in Nup50-/- gonads of both sexes suggests that NUP50 may be required for the development of PGCs at early phases prior to gonadal sex determination.
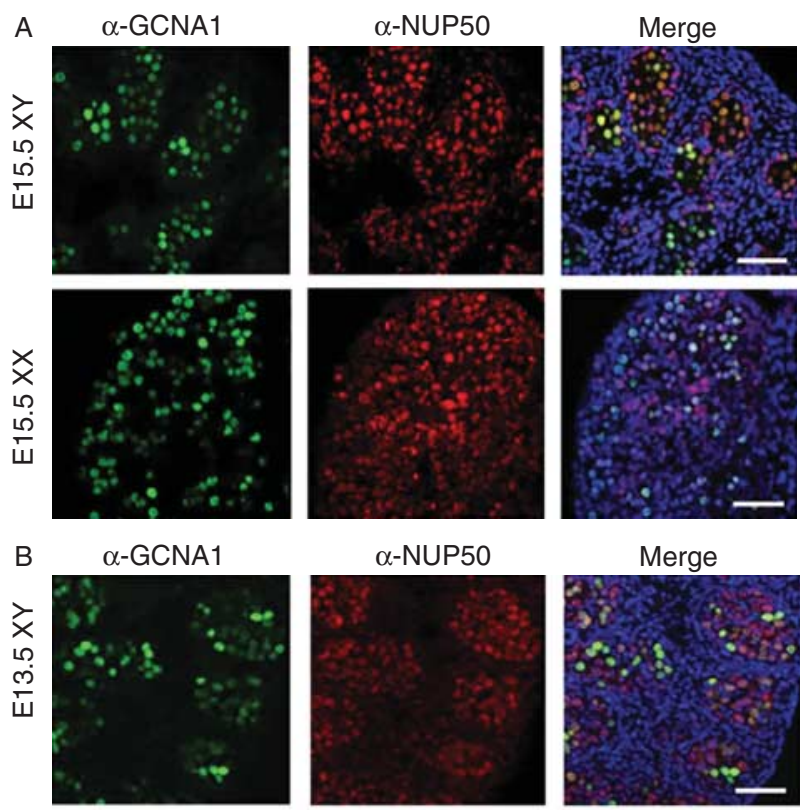

$\alpha-N U P 50$
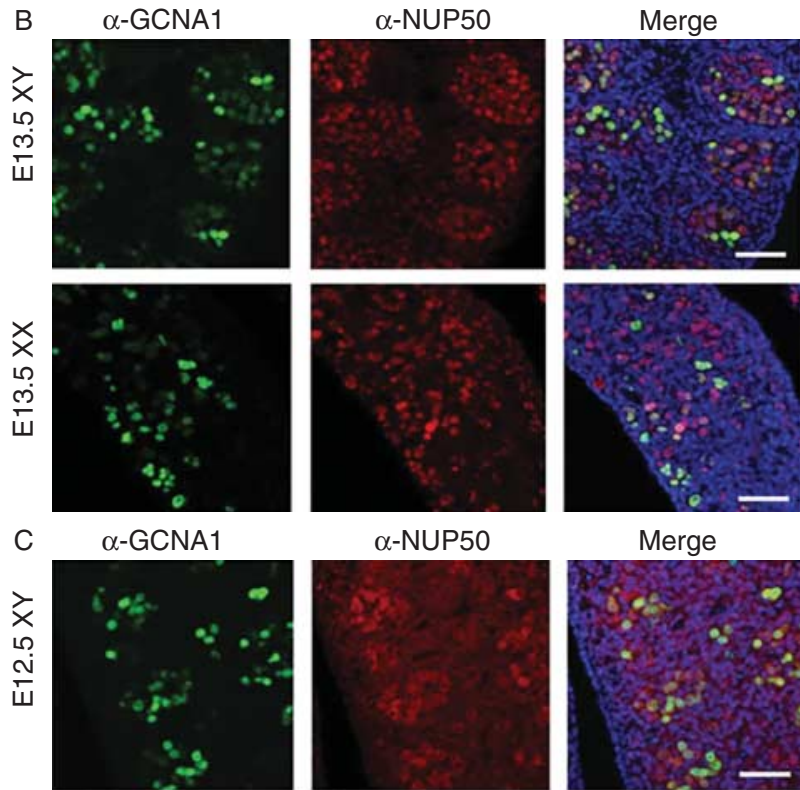

$\alpha-N U P 50$
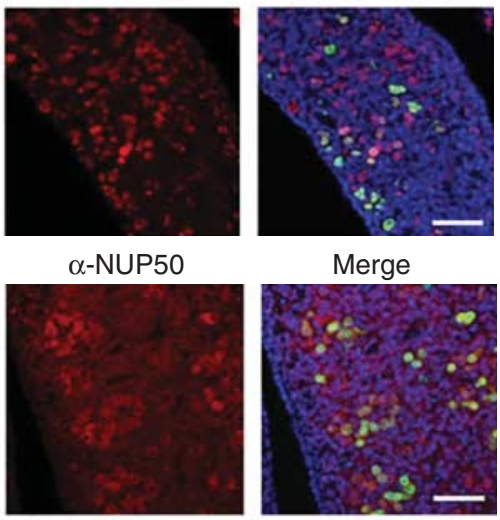

Merge
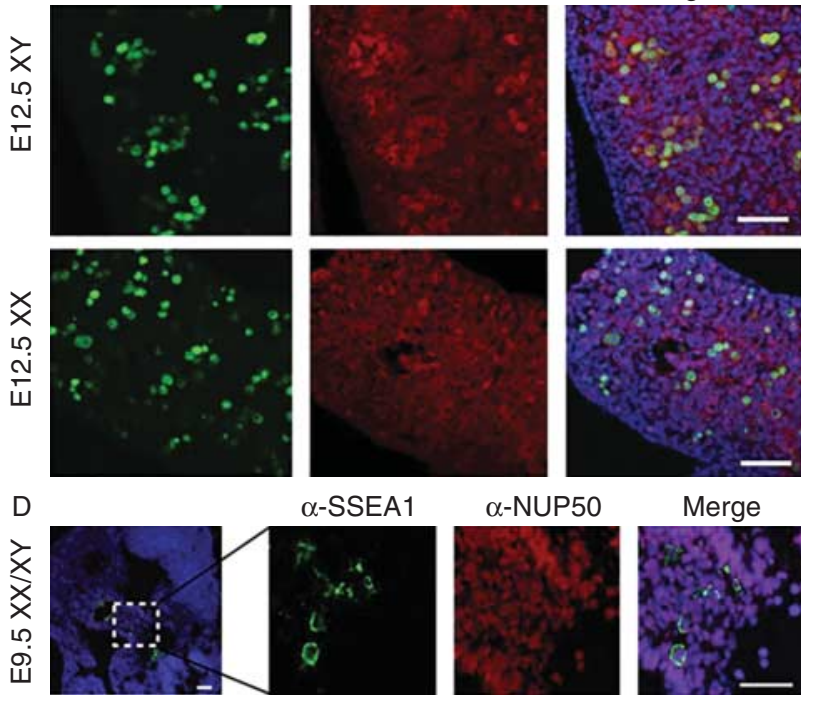

Figure 1 NUP50 expression in germ cells during embryonic development. Fluorescence staining of WT fetal gonads at E15.5 (A; $n=3)$, E13.5 (B; $n=3 \mathrm{XY}, n=4 \mathrm{XX})$, and $\mathrm{E} 12.5(\mathrm{C} ; n=3)$ was performed with anti-GCNA1 (green) and anti-NUP50 (red) antibodies and TOPRO-3 (blue). (D) Fluorescence staining of WT embryos at E9.5 was performed with anti-SSEA1 (green) and anti-NUP50 (red) antibodies, and TOPRO-3 (blue) $(n=3)$. The scale bars represent $50 \mu \mathrm{m}$. 

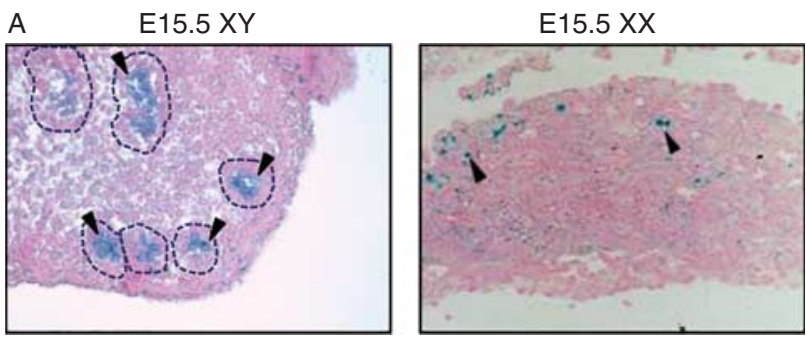

B

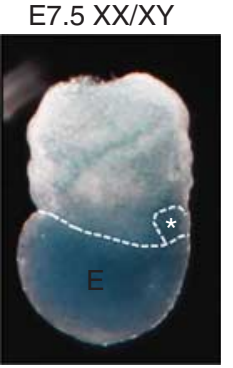

C

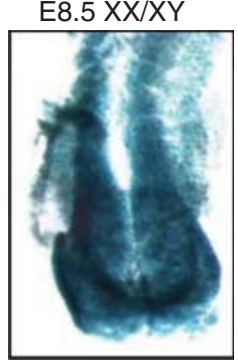

Figure 2 Identification of Nup50 expression in Nup50+/- embryos with $\beta$-gal activity. Nup50+/- fetal gonads at E15.5 (A; $n=4 \mathrm{XY}$ and $n=3 \mathrm{XX})$, embryos at E7.5 (B; $n=5)$, and E8.5 (C; $n=4)$ were stained for $\beta$-gal activity. (A) The arrowheads and the dotted lines indicate the position of germ cells and the boundary of the seminiferous tubules respectively. (B) The asterisk and ' $E$ ' indicate the areas containing PGCs and the embryo respectively. Magnification: A and B, $100 \times ; C, 400 \times$.

\section{The number of PGCs in the migratory and proliferative phases is decreased in Nup50-I- embryos}

To determine which stage of PGC development requires NUP50, we examined the populations of PGCs in Nup50-/- embryos from the specification phase to the migration phase (E7.5-E9.5). At E7.5, there was a little difference in the area staining positive for AP, which represents PGCs, between Nup50-/- and Nup50+/- embryos (Fig. 4A). At E8.5, a reduced number of PGCs was observed at the base of the allantois in Nup50-/- embryos compared with Nup50+/embryos (Fig. 4B). At E9.5, a further decrease in the number of migrating PGCs was detected along the hindgut of Nup50-/- embryos (Fig. 4C). The apparent effect of NUP50 deficiency on PGC development at E9.5 (Fig. 4C) was consistent with the expression of NUP50 in migratory PGCs at the same stage (Fig. 1D). These results suggest that NUP50 may be involved in the PGC maintenance by suppressing cell death and/or enhancing cell proliferation during the migratory and proliferative phases.

\section{Migratory PGCs exhibit a high level of apoptosis and a normal rate of proliferation in Nup50-I- embryos}

PGCs proliferate mitotically during migration to the genital ridges from E8.5 to E10.5. Various growth factors and proteins have been shown to improve the PGC survival rate and stimulate PGC proliferation (Ewen \&
A
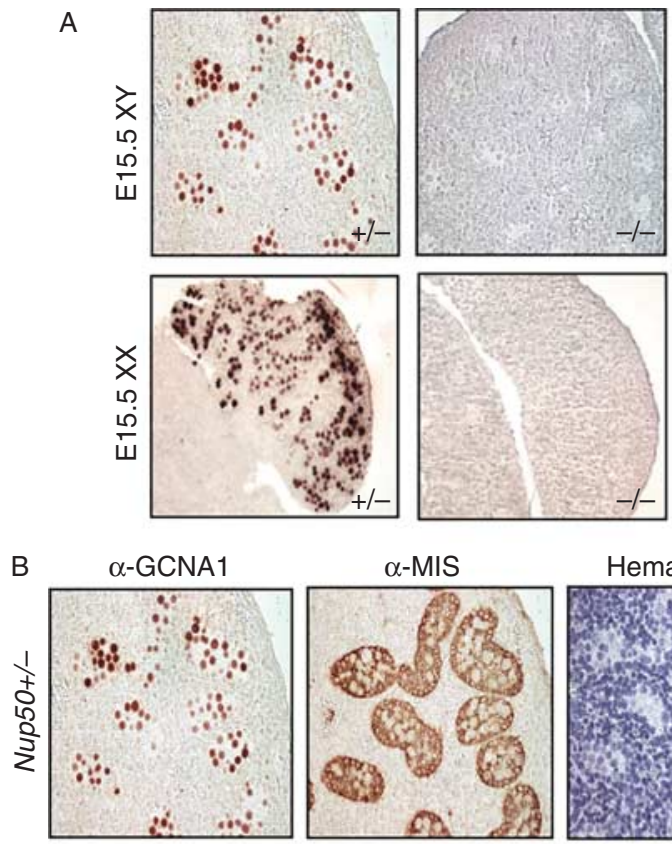

Hematoxylin
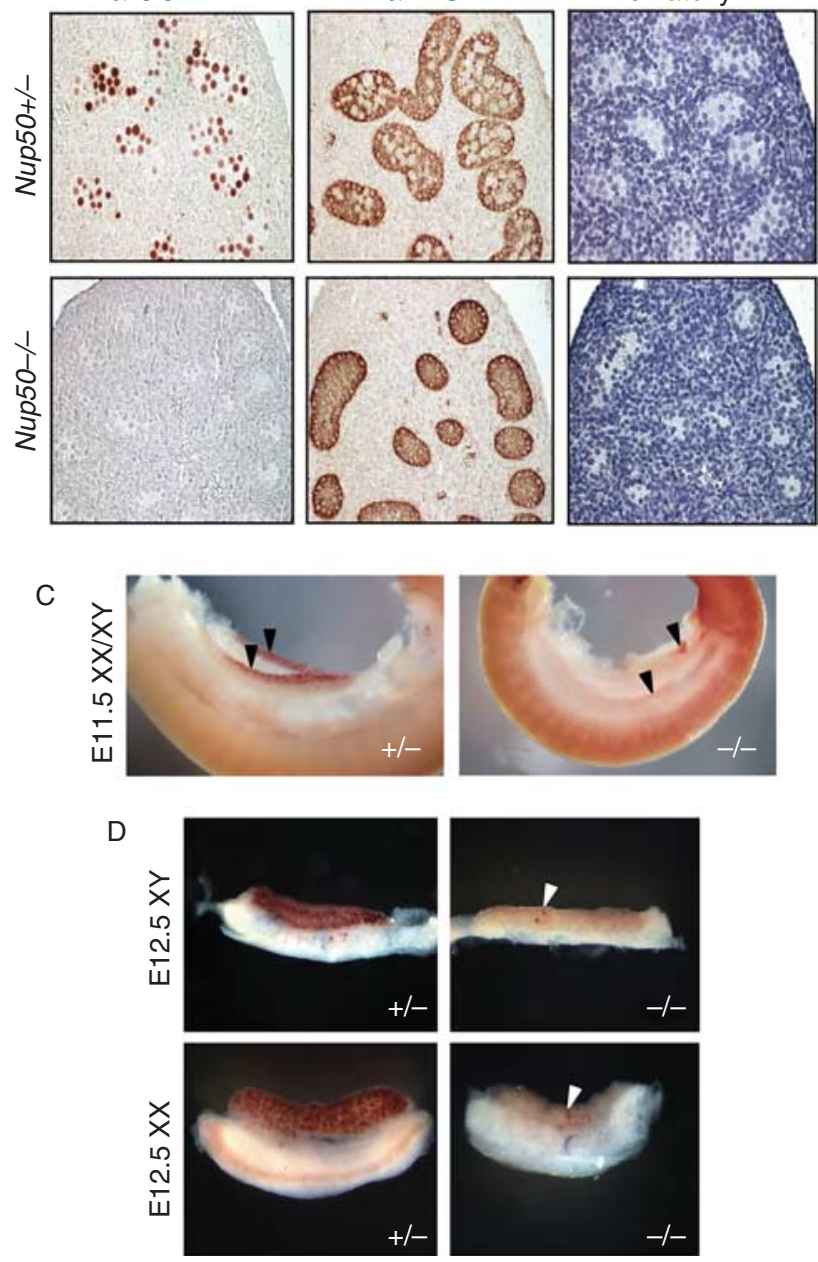

Figure 3 Impaired PGC development in Nup50-/- embryonic gonads. (A) The gonads of Nup50+/- and Nup50-/ - embryos at E15.5 were processed for IHC with anti-GCNA1 antibody $(n=3)$. (B) The testes of Nup50+/- and Nup50-/- embryos at E15.5 were processed for immunohistochemistry (IHC) with anti-GCNA1 and anti-MIS antibodies $(n=3)$. (C and D) Nup50+/- and Nup50-/- embryos at E11.5 (C; $n=6$ $+/-$ and $n=4-/-)$ and Nup50+/- and Nup50-/- gonads at E12.5 (D; $n=5 \mathrm{XY}+/-, n=3 \mathrm{XY}-/-, n=7 \mathrm{XX}+/-$, and $n=3 \mathrm{XX}-/-$ ) were stained for AP. The arrowheads indicate the positions of AP-positive PGCs. Magnification: A and B, 400×; C, 50×; D, 100×. 

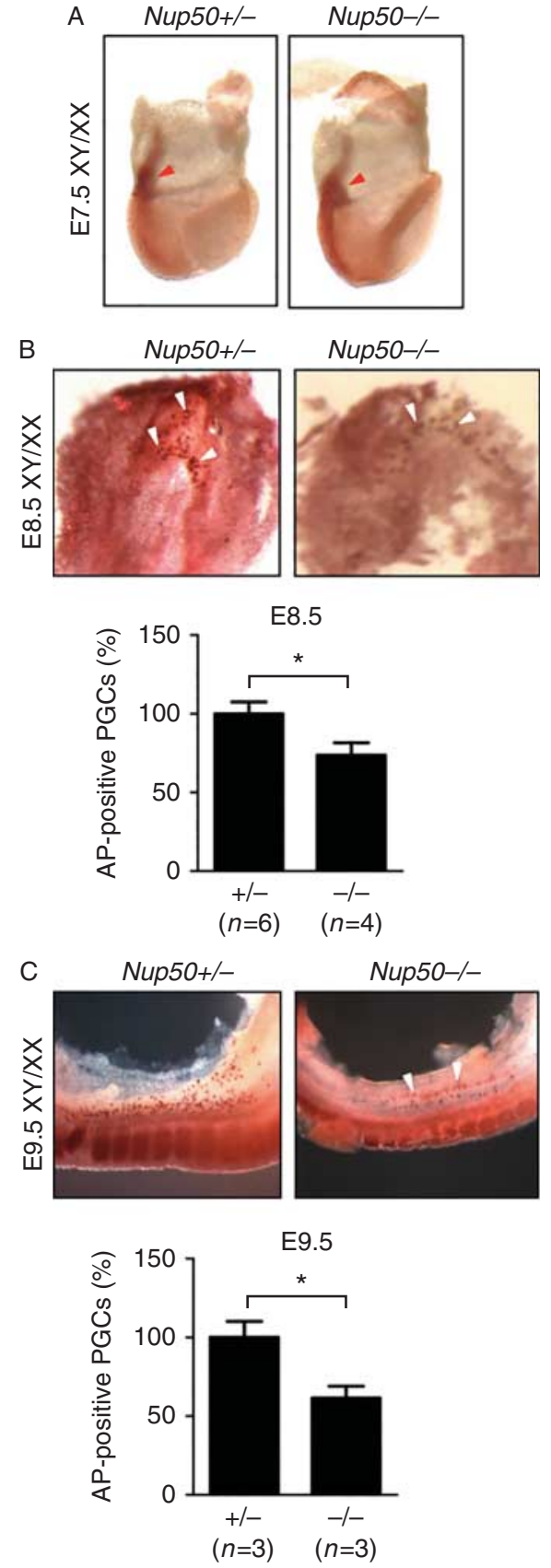

Figure 4 Reduced number of PGCs in the migratory and proliferative phases in Nup50-/- embryos. Whole-mount AP staining of Nup50+/- and Nup50-/ - embryos at E7.5 (A; $n=6+/-$ and $n=4-/-$ ), E8.5 (B; $n=6+/-$ and $n=4-/-$ ) and E9.5 (C; $n=3$ ). (B bottom and C bottom) Quantitation represents the percentage of AP-positive migrating PGCs. The arrowheads indicate the positions of AP-positive PGCs. ${ }^{*}, P<0.05$.

Koopman 2010). Therefore, we investigated whether NUP50 deficiency affects PGC survival and/or proliferation during the migratory and proliferative phases. In Nup50-/- embryos at E9.5, a subset of the PGC7-positive migratory PGCs in the hindgut was also positive for LysoTracker, an apoptosis marker (Fig. 5A).
In contrast, no PGC7-positive PGCs in Nup50+/embryos were positive for LysoTracker. Similarly, a subset of the SSEA1-positive migratory PGCs in Nup50-/- embryos but not in Nup50+/- embryos
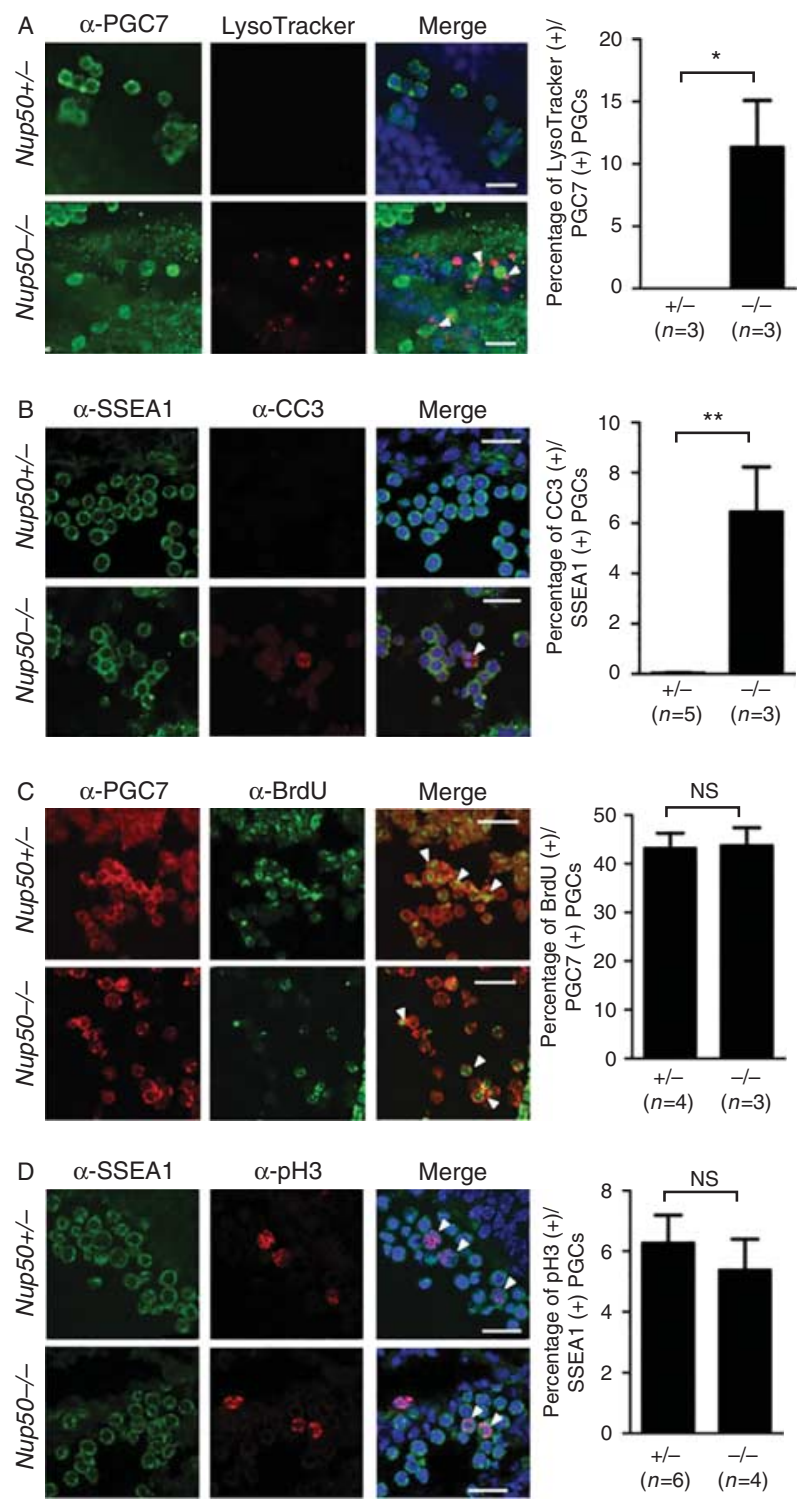

Figure 5 Increased apoptosis and normal proliferation of PGCs in Nup50-/ - embryos at E9.5. (A) Nup50+/- and Nup50-/embryos were incubated with LysoTracker (red), and then whole-mount immunofluorescence was performed with anti-PGC7 (green) antibody (left). (B) Fluorescence staining of embryos was performed with antiSSEA1 (green) and anti-cleaved caspase-3 (CC3; red) antibodies (left). (C) BrdU-incorporated cells and PGCs in embryos were detected by immunofluorescence with anti-BrdU (green), and anti-PGC7 (red) antibodies respectively (left). (D) Fluorescence staining of embryos was performed with anti-SSEA1 (green) and anti-phospho-histone $\mathrm{H} 3 \mathrm{(pH} 3$; red) antibodies. Nuclei were stained with TOPRO-3 (blue in the merged images) (left). The percentage of PGCs undergoing apoptosis or proliferating was determined by counting at least three areas or sections per embryo (right). The scale bars represent $25 \mu \mathrm{m} .{ }^{*}, P<0.05$; **, $P<0.01$; ns, not significant. 
was positive for cleaved caspase-3, another apoptosis marker (Fig. 5B).

Conversely, 5-BrdU incorporation, which marks proliferating cells, was detectable in a subset of the PGC7-positive PGCs in Nup50-/- and Nup50+/embryos at E9.5, although the number of PGC7-positive PGCs was reduced in Nup50-/- compared with Nup50+/- embryos (Fig. 5C, left). However, a quantitative analysis revealed that the PGC7-positive PGCs in Nup50+/- and Nup50-/- embryos presented similar proliferation rates (Fig. 5C, right). Similar results were obtained by analyzing the expression of phospho-histone $\mathrm{H} 3$, another proliferation marker, in SSEA1-positive PGCs in Nup50-/- and Nup50+/embryos (Fig. 5D). These results suggest that NUP50, which is expressed in PGCs at E9.5, is required for the survival but not the proliferation of migratory PGCs.

\section{Nup50-/ - embryos also show increased somatic cell apoptosis}

Because the expression of NUP50 was detected in both somatic cells and PGCs during early embryonic development (Figs 1 and 2), we examined whether NUP50 deficiency also affects the survival of somatic cells. Interestingly, some somatic cells, particularly those in the dorsal portions of the mesentery, were positive for cleaved caspase-3 in Nup50-/- embryos but not Nup50+/- embryos at E9.5 (Fig. 6A). However, phospho-histone $\mathrm{H} 3$-positive somatic cells were moderately detected in both Nup50+/- and Nup50-/embryos at E9.5 (Fig. 6B). These results suggest that NUP50 is required for the survival of somatic cells as well as migratory PGCs during this stage. The intrauterine growth retardation observed in Nup50-/- embryos (Smitherman et al. 2000) may be a consequence of the increased apoptosis of somatic cells with NUP50 deficiency.

\section{Discussion}

The expression of NUP50 is not only high in the testis but also regulated in germ cells during germ cell differentiation in adult mice (Fan et al. 1997, Smitherman et al. 2000). In the present study, we analyzed the expression of NUP50 in germ cells during embryonic development. In mouse embryos, NUP50 was highly expressed in migrating PGCs and germ cells in the gonads. Interestingly, in the adult testis, Nup50 mRNAs and NUP50 proteins were not expressed in the spermatogonial population containing stem cells of the germ cell lineage (Smitherman et al. 2000; data not shown). Therefore, the function of NUP50 in PGCs and fetal germ cells may be unrelated to the stemness of germ cells.

Several genes that are regulated during PGC specification have been identified by single-cell gene expression analysis (Saitou et al. 2002, Yabuta et al.
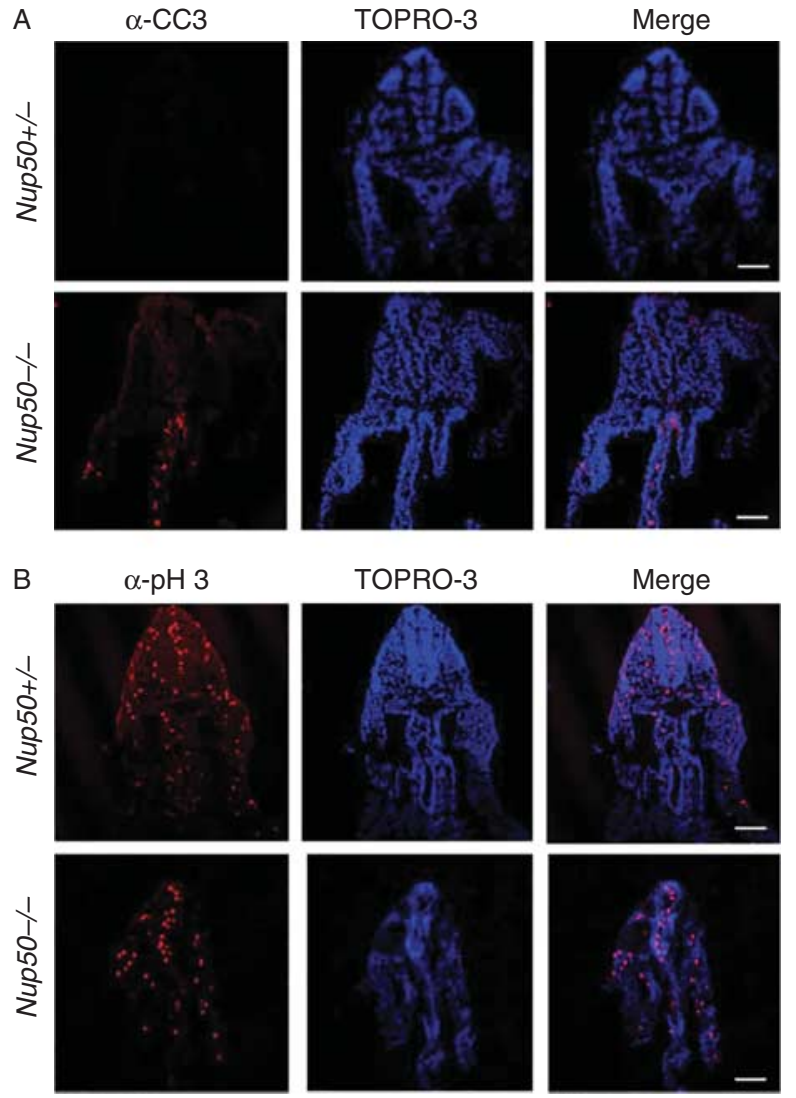

Figure 6 Somatic cells in Nup50-/- embryos undergo apoptosis. Representative sections of Nup50+/- and Nup50-/- embryos at E9.5 were stained for cleaved caspase-3 (CC3; red) $(\mathrm{A} ; n=4+/-$ and $n=3-/-$ ) or phospho-histone $\mathrm{H} 3(\mathrm{pH} 3$; red) $(\mathrm{B} ; n=4)$ and were counterstained with TOPRO-3 (blue). The scale bars represent $100 \mu \mathrm{m}$.

2006). BLIMP1, which is known as a key regulator of PGC specification, is expressed in PGCs from E7 to E13.5 (Chang et al. 2002), and its deficiency in embryos leads to a decrease in the population of PGC-like cells, resulting in the impaired proliferation and migration of PGCs (Ohinata et al. 2005). Germ cell-specific deletion of another gene product, OCT4, which is ubiquitously expressed in the embryo until E7.5 and thereafter is specifically expressed in germ cells, leads to impaired PGC specification following the loss of migrating PGCs during embryonic development (Kehler et al. 2004, Okamura et al. 2008). In this study, we demonstrated that NUP50 deficiency led to an impairment in the population of migratory PGCs at E8.5 and E9.5, the phase of PGC migration, but not at E7.5, the phase of PGC specification (Fig. 4). Therefore, we speculate that NUP50 functions during PGC migration rather than during PGC specification. Further detailed studies are necessary to address whether NUP50 is involved in PGC specification.

Notably, we observed that NUP50 deficiency in embryos appeared to impact the development of both male and female germ cells (Fig. 3). The population of 
PGCs in Nup50-/- embryos was decreased at E8.5 and further reduced at E9.5 (Fig. 4), which was likely due to an increase in apoptosis (Fig. 5). The PGC phenotypes of Nup50-/- embryos suggest that NUP50 may regulate the survival of migratory PGCs via an inhibition of apoptosis. Previous studies have identified several key genes involved in the regulation of PGC survival, which act in a manner similar to NUP50. Germ cell-specific Oct4- or Nanog-knockout embryos harbor a decreased number of PGCs, mediated by apoptosis (Kehler et al. 2004, Yamaguchi et al. 2009). In Steel-knockout embryos, the majority of PGCs show BAX-dependent apoptosis at or before E9.0, the phase of PGC migration, and no germ cells remain alive at E11.5 (Runyan et al. 2006). In addition, Nanos3-knockout embryos are characterized by a reduced number of PGCs at E12.5 due to BAX-dependent or BAX-independent apoptotic cell death (Suzuki et al. 2008). It will be interesting to investigate whether the mechanism of apoptosis in NUP50-deficient PGCs involves the BAX-dependent pathway, as has been found for other regulators of survival.

Although NUP50 had been characterized as a component of the NPC, it has been shown that several NUPs including NUP50 are involved in a variety of cellular processes such as chromatin organization and gene expression as well as nuclear transport (Kalverda et al. 2010). Based on such previous studies of NUP50 function, we carefully propose the following potential role for NUP50 in PGC development. First, NUP50 may act as a component of the NPC in PGCs as in other cells. NUP50 is required for the disassembly of import complexes of NLS-containing proteins and for the recycling of importins through interaction with importin $\alpha$ (Matsuura \& Stewart 2005). In the fetal gonad, the expression of importins including importin $\alpha 3$ and $\alpha 4$ is detected in the cytoplasm of germ cells from E12.5 to E16.5 (Hogarth et al. 2007) but has not yet been observed in germ cells at an earlier stage. As previously reported (Smitherman et al. 2000), however, protein import in Nup50-/- mouse embryonic fibroblasts was observed to be normal: several GFP-fused germ cell marker proteins, including OCT4, NANOG, and SOX2, localized normally to the nucleus (data not shown). These results suggest either no requirement for NUP50 or a redundancy in other NPC proteins in the cellular distribution of these proteins. Another possibility is that NUP50 may play an important role in PGC development via the regulation of specific genes independent of NPC function. NUP50 in Drosophila is able to regulate the expression of developmental genes through a direct interaction with chromosomes inside of the nucleoplasm (Kalverda et al. 2010).

In summary, we demonstrate that NUP50 is expressed in PGCs and fetal germ cells of both sexes. Moreover, NUP50 deficiency causes apoptosis of PGCs during their migration into genital ridges, resulting in limited numbers of PGCs in the fetal gonads. Taken together, these data indicate that NUP50 is required for the survival of PGCs.

\section{Declaration of interest}

The authors declare that there is no conflict of interest that could be perceived as prejudicing the impartiality of the research reported.

\section{Funding}

This research was supported by the Basic Science Research Program through the National Research Foundation of Korea (NRF) funded by the Ministry of Education, Science and Technology (NRF-2014R1A4A1003642 and NRF-2014R1 A2A1A11051396).

\section{Acknowledgements}

We thank Dr George C Enders (University of Kansas Medical Center, KS) and Dr Toshinobu Nakamura (Osaka University, Japan) for kindly providing us anti-GCNA1 and anti-PGC7 antibodies respectively.

\section{References}

Agulnik Al, Mitchell MJ, Mattei MG, Borsani G, Avner PA, Lerner JL \& Bishop CE 1994 A novel X gene with a widely transcribed Y-linked homologue escapes $\mathrm{X}$-inactivation in mouse and human. Human Molecular Genetics 3 879-884. (doi:10.1093/hmg/3.6.879)

Anderson R, Copeland TK, Scholer H, Heasman J \& Wylie C 2000 The onset of germ cell migration in the mouse embryo. Mechanisms of Development 91 61-68. (doi:10.1016/S0925-4773(99)00271-3)

Chang DH, Cattoretti G \& Calame KL 2002 The dynamic expression pattern of B lymphocyte induced maturation protein-1 (Blimp-1) during mouse embryonic development. Mechanisms of Development 117 305-309. (doi:10.1016/S0925-4773(02)00189-2)

Dolci S, Williams DE, Ernst MK, Resnick JL, Brannan $\mathrm{Cl}$, Lock LF, Lyman SD, Boswell HS \& Donovan PJ 1991 Requirement for mast cell growth factor for primordial germ cell survival in culture. Nature $\mathbf{3 5 2}$ 809-811. (doi:10.1038/352809a0)

Doye V \& Hurt E 1997 From nucleoporins to nuclear pore complexes. Current Opinion in Cell Biology 9 401-411. (doi:10.1016/S09550674(97)80014-2)

Ewen KA \& Koopman P 2010 Mouse germ cell development: from specification to sex determination. Molecular and Cellular Endocrinology 323 76-93. (doi:10.1016/j.mce.2009.12.013)

Fan F, Liu CP, Korobova O, Heyting C, Offenberg HH, Trump G \& Arnheim N 1997 cDNA cloning and characterization of Npap60: a novel rat nuclear pore-associated protein with an unusual subcellular localization during male germ cell differentiation. Genomics 40 444-453. (doi:10.1006/geno.1996.4557)

Ginsburg M, Snow MH \& McLaren A 1990 Primordial germ cells in the mouse embryo during gastrulation. Development 110 521-528.

Hogarth CA, Jans DA \& Loveland KL 2007 Subcellular distribution of importins correlates with germ cell maturation. Developmental Dynamics 236 2311-2320. (doi:10.1002/dvdy.21238)

Kalverda B, Pickersgill H, Shloma VV \& Fornerod M 2010 Nucleoporins directly stimulate expression of developmental and cell-cycle genes inside the nucleoplasm. Cell 140 360-371. (doi:10.1016/j.cell.2010.01.011)

Kehler J, Tolkunova E, Koschorz B, Pesce M, Gentile L, Boiani M, Lomeli H, Nagy A, McLaughlin KJ, Scholer HR et al. 2004 Oct4 is required for primordial germ cell survival. EMBO Reports 5 1078-1083. (doi:10. 1038/sj.embor.7400279) 
Lindsay ME, Plafker K, Smith AE, Clurman BE \& Macara IG 2002 Npap60/Nup50 is a tri-stable switch that stimulates importin- $\alpha$ : $\beta$-mediated nuclear protein import. Cell 110 349-360. (doi:10.1016/ S0092-8674(02)00836-X)

Matsuura Y \& Stewart M 2005 Nup50/Npap60 function in nuclear protein import complex disassembly and importin recycling. EMBO Journal 24 3681-3689. (doi:10.1038/sj.emboj.7600843)

McLaren A 2003 Primordial germ cells in the mouse. Developmental Biology 262 1-15. (doi:10.1016/S0012-1606(03)00214-8)

Nagy A 2003 In Manipulating the Mouse Embryo: A Laboratory Manual. Cold Spring Harbor, NY: Cold Spring Harbor Laboratory Press.

Ohinata Y, Payer B, O'Carroll D, Ancelin K, Ono Y, Sano M, Barton SC, Obukhanych T, Nussenzweig M, Tarakhovsky A et al. 2005 Blimp1 is a critical determinant of the germ cell lineage in mice. Nature 436 207-213. (doi:10.1038/nature03813)

Okamura D, Tokitake Y, Niwa H \& Matsui Y 2008 Requirement of Oct3/4 function for germ cell specification. Developmental Biology 317 576-584. (doi:10.1016/j.ydbio.2008.03.002)

Resnick JL, Bixler LS, Cheng L \& Donovan PJ 1992 Long-term proliferation of mouse primordial germ cells in culture. Nature 359 550-551. (doi:10. 1038/359550a0)

Runyan C, Schaible K, Molyneaux K, Wang Z, Levin L \& Wylie C 2006 Steel factor controls midline cell death of primordial germ cells and is essential for their normal proliferation and migration. Development 133 4861-4869. (doi:10.1242/dev.02688)

Saitou M, Barton SC \& Surani MA 2002 A molecular programme for the specification of germ cell fate in mice. Nature 418 293-300. (doi:10. 1038/nature00927)

Smitherman M, Lee K, Swanger J, Kapur R \& Clurman BE 2000 Characterization and targeted disruption of murine Nup50, a p27(Kip1)-interacting component of the nuclear pore complex. Molecular and Cellular Biology 20 5631-5642. (doi:10.1128/MCB.20.15.56315642.2000)
Suzuki H, Tsuda M, Kiso M \& Saga Y 2008 Nanos3 maintains the germ cell lineage in the mouse by suppressing both Bax-dependent and independent apoptotic pathways. Developmental Biology 318 133-142. (doi:10.1016/j.ydbio.2008.03.020)

Tam PP \& Snow MH 1981 Proliferation and migration of primordial germ cells during compensatory growth in mouse embryos. Journal of Embryology and Experimental Morphology 64 133-147.

Tsuda M, Sasaoka Y, Kiso M, Abe K, Haraguchi S, Kobayashi S \& Saga Y 2003 Conserved role of nanos proteins in germ cell development. Science 301 1239-1241. (doi:10.1126/science.1085222)

Weidinger G, Stebler J, Slanchev K, Dumstrei K, Wise C, Lovell-Badge R, Thisse C, Thisse B \& $\boldsymbol{R a z}$ E 2003 dead end, a novel vertebrate germ plasm component, is required for zebrafish primordial germ cell migration and survival. Current Biology 13 1429-1434. (doi:10.1016/S0960-9822 (03)00537-2)

Yabuta Y, Kurimoto K, Ohinata Y, Seki Y \& Saitou M 2006 Gene expression dynamics during germline specification in mice identified by quantitative single-cell gene expression profiling. Biology of Reproduction 75 705-716. (doi:10.1095/biolreprod.106.053686)

Yamaguchi S, Kurimoto K, Yabuta Y, Sasaki H, Nakatsuji N, Saitou M \& Tada T 2009 Conditional knockdown of Nanog induces apoptotic cell death in mouse migrating primordial germ cells. Development 136 4011-4020. (doi:10.1242/dev.041160)

Yao HH, Whoriskey W \& Capel B 2002 Desert Hedgehog/Patched 1 signaling specifies fetal Leydig cell fate in testis organogenesis. Genes and Development 16 1433-1440. (doi:10.1101/gad.981202)

Received 10 December 2014

First decision 23 February 2015

Revised manuscript received 20 October 2015

Accepted 29 October 2015 